\title{
A New Method to Determine the Skin Thickness of Asymmetric UF-Membranes Using Colloidal Gold Particles
}

\author{
FOLKERT PETRUS CUPERUS, ${ }^{1}$ DERK BARGEMAN, AND KEES SMOLDERS \\ Department of Chemical Technology, University of Twente, P.O. Box 217, 7500 AE Enschede, The Netherlands
}

Received April 27, 1989; accepted July 11, 1989

\begin{abstract}
In this paper a new method is presented for the determination of the skin thickness of asymmetric ultrafiltration membranes. The method is based on the use of well-defined, uniformly sized colloidal gold particles, permeated from the sublayer side of the membrane, combined with electron microscopic analysis of the membrane afterward. Using this method poly(2,6-dimethyl-1,4-phenylene oxide) (PPO) and polysulfone (PSf) membranes were investigated. PPO membranes appeared to have a well-defined skin layer with a thickness of about $0.2 \mu \mathrm{m}$ and a pore size distinctly different from that in the macroporous layer underneath. In the case of PSf such a distinct skin layer cannot be defined. The size of the pores in these membranes gradually increases from skin to sublayer. 1990 Academic Press, Inc.
\end{abstract}

\section{INTRODUCTION}

The application of ultrafiltration (UF) in industrial processes has gained an increased interest in recent years. In most of these separation processes asymmetric membranes are used. Asymmetric membranes consist of a thin, relatively dense top layer responsible for the separation characteristics of the membrane and an open sublayer that is supposed not to influence the membrane performance. The size distribution of the pores present in the skin determines the selectivity of the membrane, whereas the skin thickness is an important parameter when hydraulic permeability of the membrane is concerned.

Pore-size distributions of asymmetric UF membranes can be measured using several independent methods such as thermoporometry, gas adsorption-desorption hysteresis, bubble pressure measurements, and permporometry (1-3). These sometimes quite sophisticated methods allow the measurement of pore sizes in the range of several nanometers to tens of nanometers. Furthermore, gas and liquid permeation measurements are used extensively

\footnotetext{
${ }^{1}$ To whom correspondence should be addressed.
}

(4-6). When a combination of these techniques is used, a reasonable insight into the pore morphology of the membrane is possible.

For a better understanding of the relation between membrane structure and transport through the membrane, however, more knowledge of another crucial parameter, the skin thickness of an asymmetric UF membrane, is required. Although there are many different methods available for measuring pore size or pore-size distribution, there are only a few methods known for measuring the skin thickness of asymmetric porous UF membranes. In most cases the skin thickness of the membrane is estimated by means of scanning electron microscopy (SEM). The size of the pores in the skin, however, is too small to be distinguished with SEM and often a certain gradient in pore size from the top of the skin toward the porous sublayer exists. In the case of asymmetric membranes made by phase inversion the determination of the skin thickness is therefore hardly possible and the analysis is limited to a rough estimation.

Another possibility for obtaining information about the thickness of the skin layer is to calculate it from pure water fluxes or from pore-size/pore-volume distributions as mea- 
sured by, for example, thermoporometry (7). Here the assumption of a pore model is necessary and the final result depends strongly on the model used.

The new method presented here is based on the penetration of colloidal particles of wellknown size and very narrow size distribution into the "open" macroporous sublayer of an asymmetric UF membrane. A monodisperse colloidal gold solution, existing of particles with a size slightly larger than the size of the pores present in the skin, is filtered in the reverse way compared to normal filtration. The particles penetrate in the porous support until small pores near or in the skin are reached. When the particles used are only slightly larger than the pores in the skin, a thin layer not permeated by colloids is formed and can be distinguished by means of SEM. By comparing experiments done with different colloidal solutions containing particles of an increasing size, one can obtain information about the pore size in the skin and sublayer.

\section{EXPERIMENTAL}

\section{Preparation and Analysis of Gold Colloids}

The synthesis of monodisperse colloidal gold particles is described in the literature (8, 9). The sizes of these particles are in the 5-50 $\mathrm{nm}$ (diameter) range and are dependent on the conditions during synthesis. In this study two sols with different particle sizes were used.

Colloidal solutions containing particles with a mean diameter of $6 \mathrm{~nm}$ were obtained in the following way: $10.0 \mathrm{ml}$ of a $1.0 \mathrm{wt} \%$ solution of chloroauric acid $\left(\mathrm{HAuCl}_{4}\right)$ was added to $1000 \mathrm{ml}$ water (filtered by reverse osmosis) and the $\mathrm{pH}$ of the solution was adjusted to a value of 7.2 with $0.1 \mathrm{MK}_{2} \mathrm{CO}_{3}$ solution. The solution was heated to boiling in a reaction vessel and $10.0 \mathrm{ml}$ diethyl ether saturated with white phosphorus (at $20^{\circ} \mathrm{C}$ ) was added to the well-stirred solution. Then the solution was cooled down and a stream of air was lead through the deep red solution during one night to remove the excess unreacted phosphorus.

Sols containing particles with a mean di- ameter of $50 \mathrm{~nm}$ were made by adding a 10.0 $\mathrm{ml} 1.0 \mathrm{wt} \%$ sodium citrate solution to a boiling solution of $1000 \mathrm{ml}$ water (purified by reverse osmosis) and $10.0 \mathrm{ml} 1.0 \mathrm{wt} \%$ chloroauric acid. During the reaction the solution was stirred vigorously until the color was red. After $5 \mathrm{~min}$ the heating was stopped and the solution was cooled down.

All chemicals used were of analytical grade and supplied by Merck.

The sols were characterized by transmission electron microscopy. Sol droplets were sprayed on coal films and after $1 \mathrm{~min}$ the excess liquid was removed carefully by dipping with a filter paper. Transmission micrographs were made using a Jeol 200 CT device. The resolution of this apparatus is better than $0.5 \mathrm{~nm}$. Particlesize distributions were determined by counting particles and by measurement of their cross section by means of image processing.

\section{Membrane Preparation and Permeation}

Poly(2,6-dimethyl-1,4-phenylene oxide) (PPO) and polysulfone (PSf) membranes were investigated. PPO membranes were prepared from a $10 \mathrm{wt} \%$ polymer solution in a mixture of trichloroethylene and octanol-1 in a weight ratio $78 / 22$. PSf membranes were made from a $15 \mathrm{wt} \%$ PSf solution in DMF. In both cases the solutions were cast at room temperature to a thickness of $0.20 \mathrm{~mm}$ on a glass plate. The PPO films were coagulated in a methanol bath and the PSf films in a water bath. All solvents used were of analytical grade.

The membranes were placed with the skin side down in an Amicon cell (membrane area, $38.5 \mathrm{~cm}^{2}$ ) and permeated at 1 bar with $\mathrm{RO}-$ water for at least $30 \mathrm{~min}$. Then a small amount of the dark red colloidal gold solution was added to enter the membrane from the porous side. The colloidal gold solution, containing $100 \mathrm{ppm}$ gold, was permeated through the membrane at 1 bar. In all cases a colorless permeate was obtained, indicating that retention was at least $99 \%$. The amount of solution used was adjusted to obtain a detectable layer in the membrane: in the case of 6-nm particles 
$100 \mathrm{ml}$ sol solution was needed, and in the case of $50-\mathrm{nm}$ particles $200 \mathrm{ml}$ sol solution was needed. For each membrane only one type of colloidal solution, with 6- or 50-nm particles, was used. The experiments were performed in dead-end filtration and diafiltration modes. Results were identical for both types of filtration.

\section{Scanning Electron Microscopy}

Cross section electron micrographs were obtained with a Jeol JSM 35 CF scanning electron microscope using both the secondary electron image (SEI) and the backscattered electron image (BEI) modes. Micrographs made in the SEI mode are essentially topographical, and the rough morphological structure can be examined. The backscattered mode yeilds not only topographical but also analytical information about the specimen. In this mode the contrast is dependent on the atomic number of the materials present in the sample. In our case the presence of gold particles causes light areas in the dark polymer matrix.

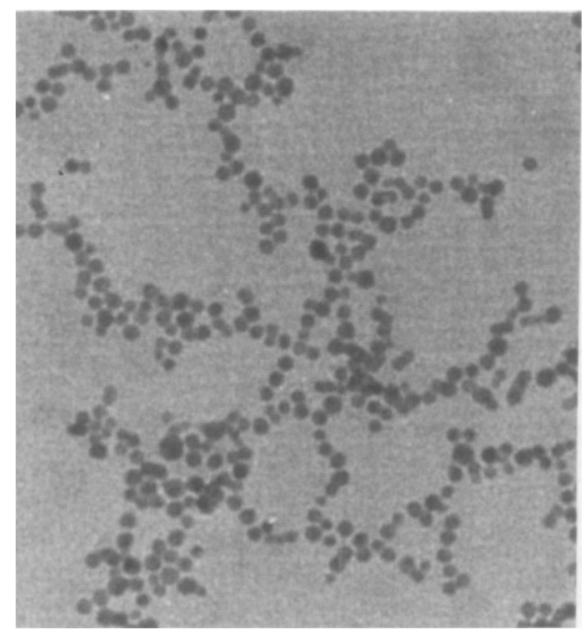

(a)

$$
100 \mathrm{~nm}
$$

Dry membrane samples were prepared by immersion of the wet membrane for $15 \mathrm{~min}$ in a 50:50 (w/w) ethanol/water bath and another $15 \mathrm{~min}$ in a pure ethanol bath, after which the membrane was dried at room temperature in a vacuum chamber. A thin gold layer $(\sim 30 \mathrm{~nm})$ was sputtered on top of the membrane. Again the pores of the membrane were filled with ethanol and fractured at liquid nitrogen temperature. Only in this way a cross section with a sharp fracture is obtained. The sample was dried as described before. To prevent the sample from becoming charged during electron microscopy, it was covered with a coal layer by using a Balzers BSV 202 coal evaporation unit.

As a result of the entire procedure a sandwich structure is formed, consisting of a "diffuse" gold layer that results from the permeated particles, a sputtered gold layer on top of the membrane, and an impenetrable skin layer in between.

\section{RESULTS}

TEM micrographs of the colloidal solutions are given in Figs. 1a and 1b. Figure 2 shows

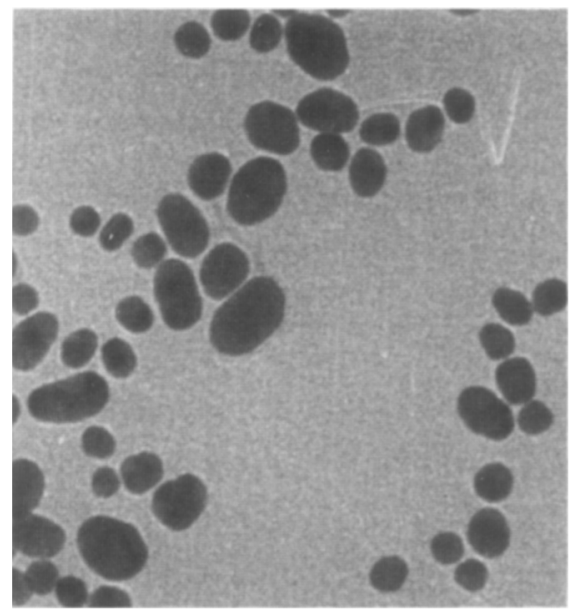

(b)

\section{$100 \mathrm{~nm}$}

FIG. 1. Typical transmission electron micrographs of colloid gold solutions. (a) Mean diameter, $\langle d\rangle=6$ $\mathrm{nm}$; (b) mean diameter, $\langle d\rangle=50 \mathrm{~nm}$. 

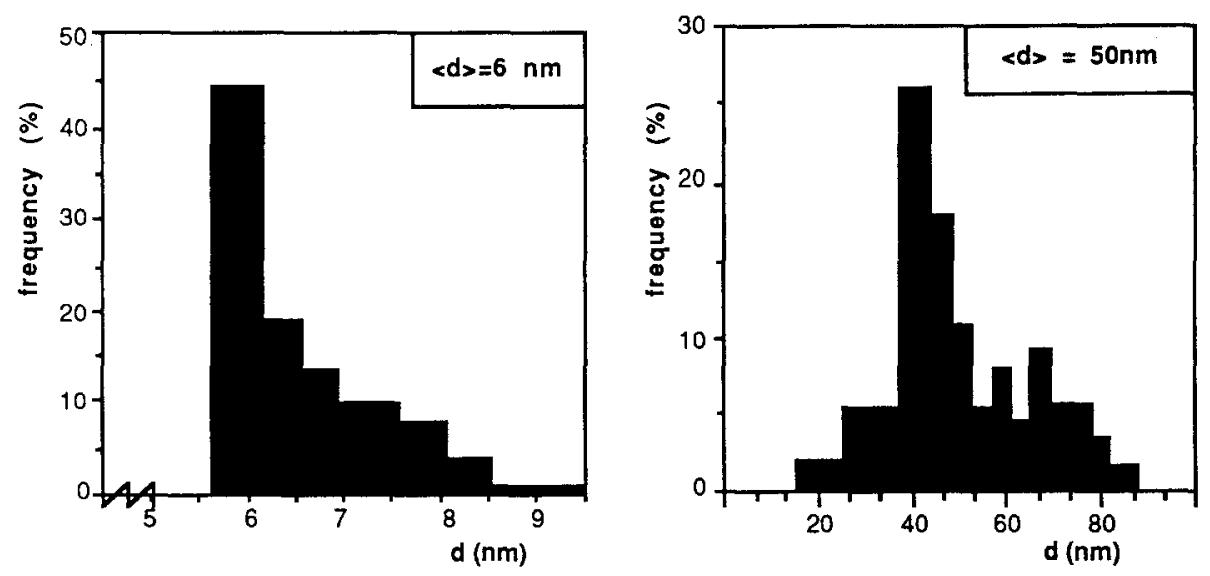

FIG. 2. Particle-size distributions of gold colloids. In the case of $\langle d\rangle=6 \mathrm{~nm}$, the size of 500 particles was evaluated; in the case of $\langle d\rangle=50 \mathrm{~nm}, 300$ particles were measured.

the particle-size distributions of the sols. The distribution of the sol with the smaller particles is asymmetric and very sharp; $72 \%$ of the particles have diameters between 5.6 and $6.4 \mathrm{~nm}$. Particles with diameters smaller than $5.6 \mathrm{~nm}$ are not present. The sol with the larger particles has a more Gaussian distribution. In this case the mean particle diameter is $50 \mathrm{~nm}$ with a standard deviation of $5 \mathrm{~nm}$.

Figures 3 and 4 show the cross sections of the permeated PPO and PSf membranes, where $a$ and $b$ refer to the two sols used (diameters, 6 and $50 \mathrm{~nm}$, respectively) for each polymer. For the examination of these cross sections, the electron microscope is used in the backscattered and "the normal" secondary electron image modes. The right-hand side of Figs. 3 and 4 shows the cross sections of the membranes in the SEI mode. From these pictures it can be seen that the samples are sharp fractured, as is necessary for proper analysis and interpretation. In the backscattered mode the presence of gold particles can be clearly detected and although the sol particles cannot be seen one by one, the edge formed by the permeated particles can be distinguished easily. From the backscattered image (BEI, lefthand side of Figs. 3 and 4), three layers can be recognized: a thin light line caused by the sputtered gold layer on top, a more diffuse layer caused by the penetrated gold particles, and in between the two lighter areas is the impermeable (dark) layer in which no particles are present since the pore size is smaller than the particle size of the sol.

Using a sol with particle size of $6 \mathrm{~nm}$ for both membranes, an impenetrable layer with a thickness of about $0.2 \mu \mathrm{m}$ is detected. Although the boundary of the permeated gold particles is somewhat meandering, the thickness of the impermeable layer is defined quite well, showing a skin thickness which is of the same order for PPO and PSf membranes, $0.2 \mu \mathrm{m}$.

When a sol with larger particle size is used the two membranes behave in a different way. For PSf membranes a larger thickness of the impermeable layer is found, from $0.2 \mu \mathrm{m}$ for 6-nm sols to $0.4-0.5 \mu \mathrm{m}$ for $50-\mathrm{nm}$ sols (Fig. 4b) while the layer still is uniform in thickness.

When a PPO membrane is treated with a sol containing particles of $50 \mathrm{~nm}$ (Fig. 3b) the top layer looks somewhat more frayed and the thickness varies between 0.2 and $0.3 \mu \mathrm{m}$. This increase in thickness is very small compared to the situation where $6-\mathrm{nm}$ particles were used.

We also performed experiments where more sol solution was used $(1000 \mathrm{ml})$. In this case the macrovoids just beneath the skin appeared to be filled with gold. This indicates that the macrovoids take care of a major part of the 

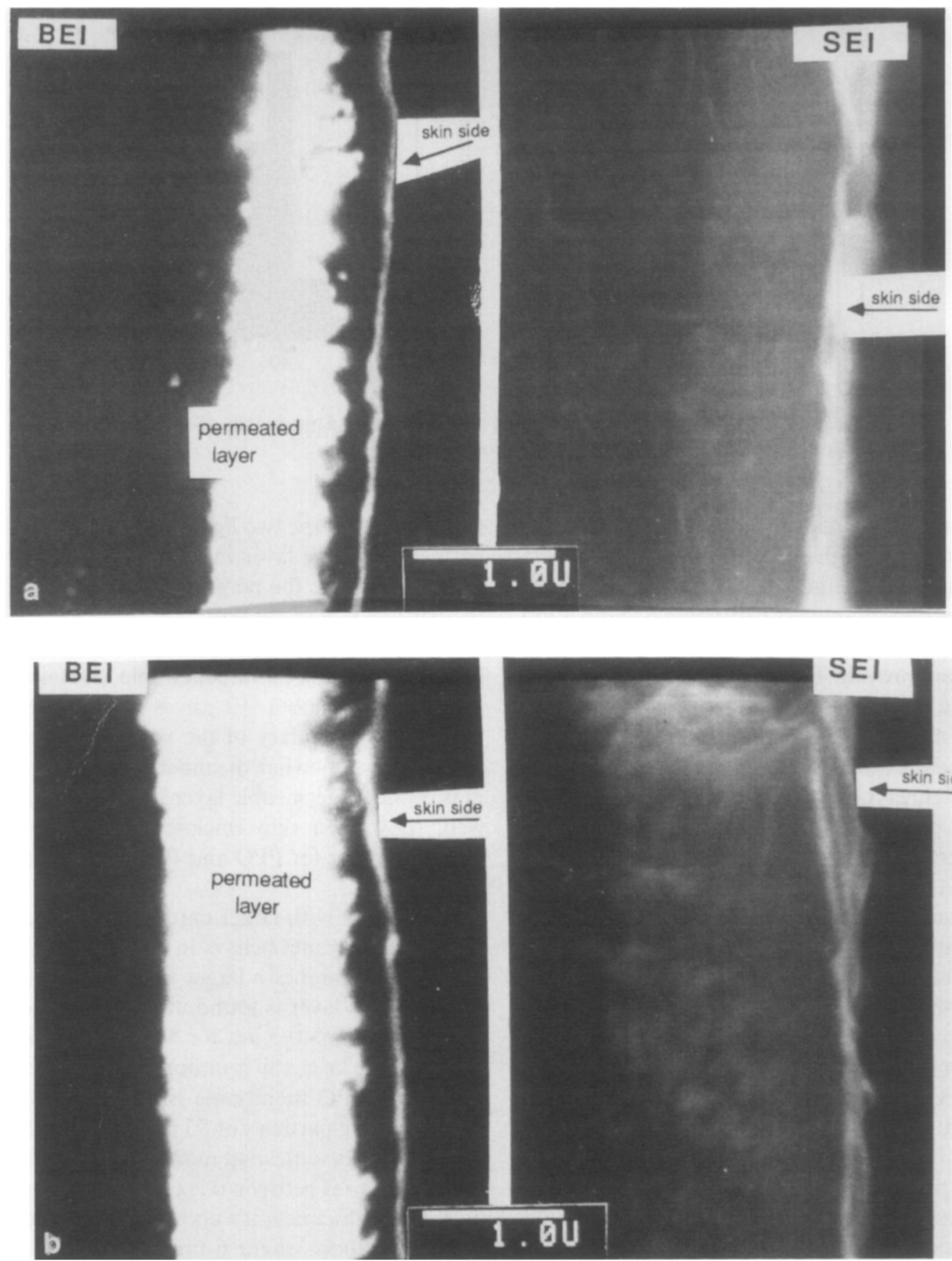

skin side


. 

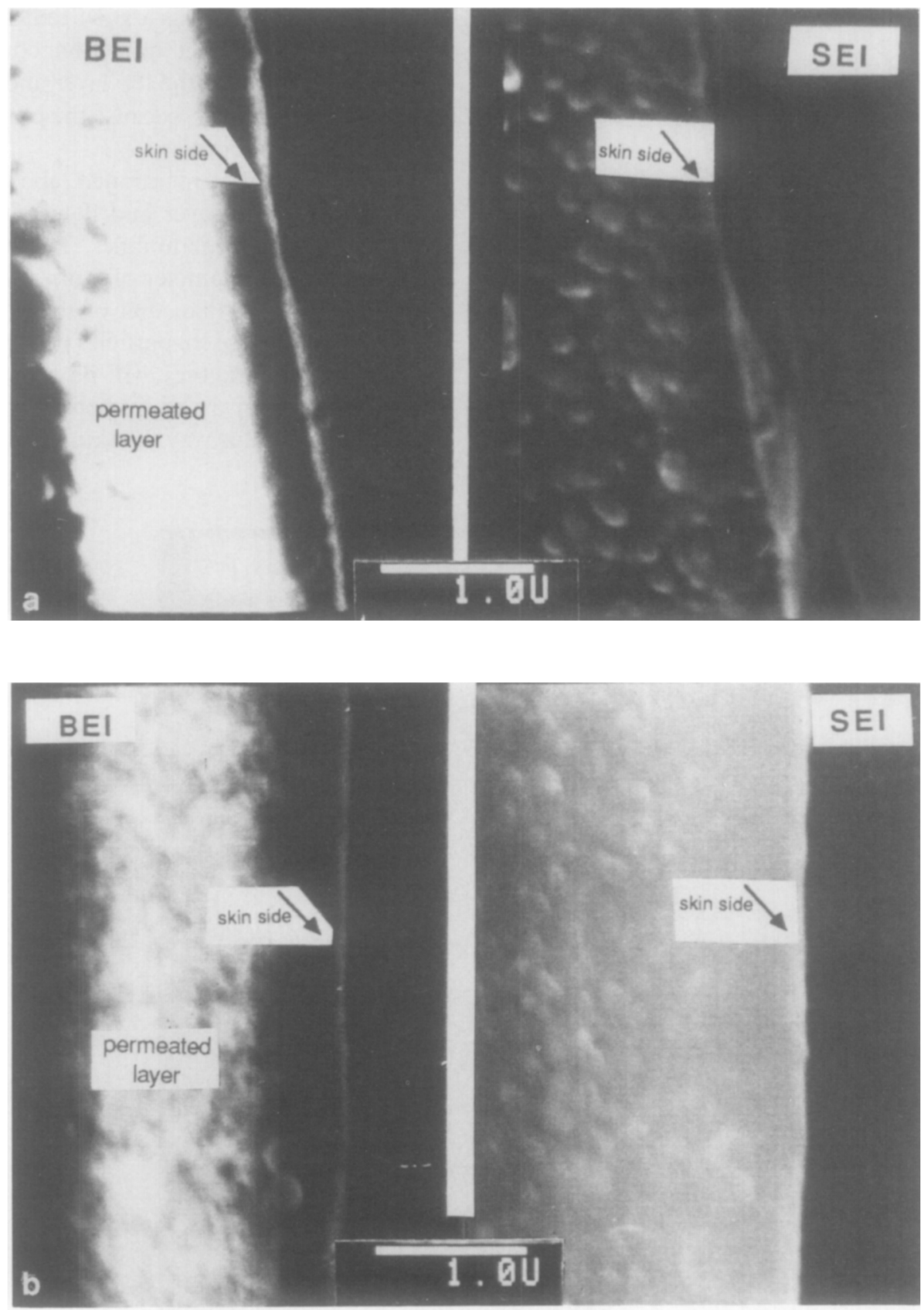

FIG. 4. (a) Cross section of a PSf-membrane, using BEI (left-hand side) and SEI (right-hand side) mode, permeated with gold sol solution; mean colloid particle diameter, $6 \mathrm{~nm}$. The cross section of the membrane has been tilted slightly to make the sputtered layer more visible. (b) Cross section of a PSf membrane, using BEI (left-hand side) and SEI (right-hand side) modes, permeated with gold sol solution; mean colloid particles diameter, $50 \mathrm{~nm}$. 
transport in the sublayer of the membrane (Fig. 5).

\section{DISCUSSION}

Similarly prepared PPO and PSf membranes previously $(3,7)$ have been characterized by thermoporometry and gas adsorptiondesorption hysteresis. It was found that PPO membranes had a very narrow pore-size distribution, with a mean pore diameter of 4.5 $\mathrm{nm}$. The pore-size distribution of PSf appeared to be rather broad, but thermoporometry showed that the smallest pores had a diameter of about $5 \mathrm{~nm}$ (3). It was argued that for PSf membranes no distinct transition could be seen between pores in the top layer and those in the supporting layer, because the pore sizes exhibit a gradual increase.

The small pores, mentioned above, are present in the skin layer and determine the performance of the membranes. The sol particles used have a diameter of $6 \mathrm{~nm}$, which is only slightly larger than that of the pores in the skin. When these sol particles penetrate in the porous support, they will be resisted by pores smaller than $6 \mathrm{~nm}$, present in or very near the skin. In this way the skin layer thick-

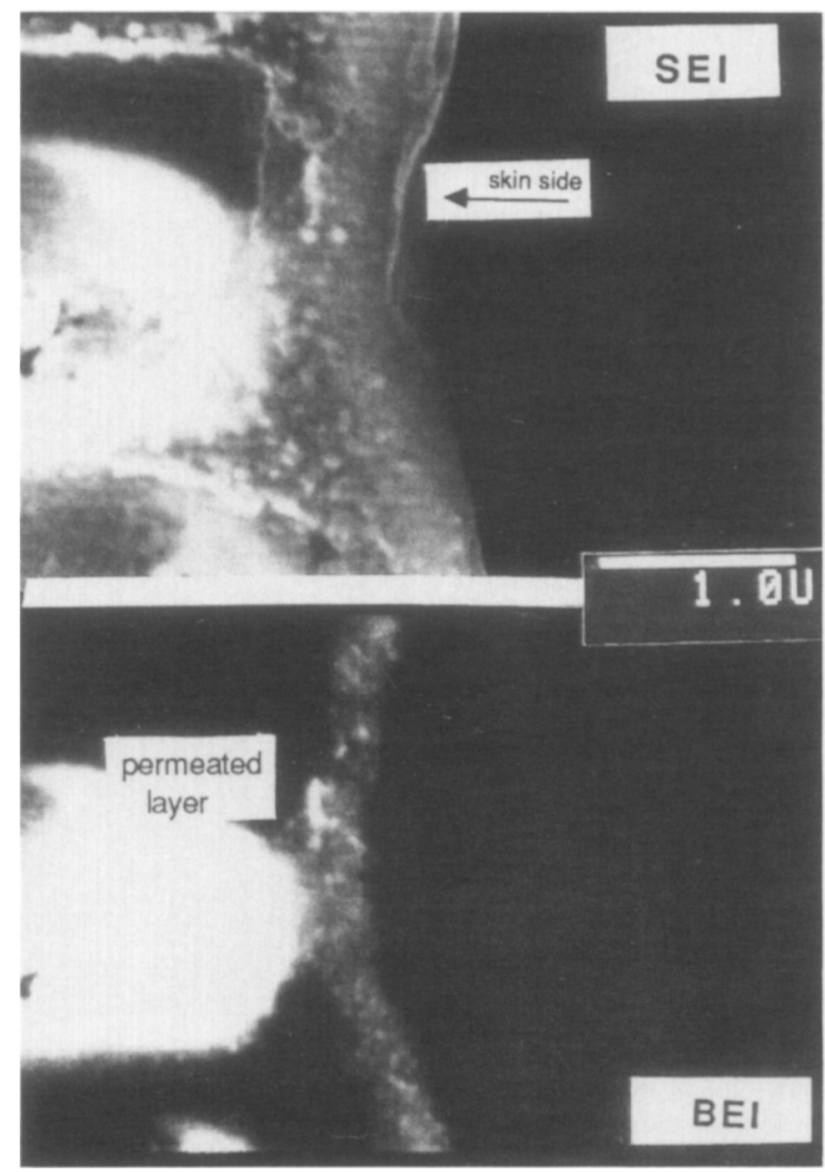

FIG. 5. Cross section of a PPO membrane using SEI (top) and BEI (bottom) modes. In this case the membrane was permeated with $1000 \mathrm{ml}$ gold sol solution; mean particle diameter, $6 \mathrm{~nm}$. No gold layer was sputtered on top of the membrane, but the position of the membrane on the top (SEI) and the bottom (BEI) of the figure is exactly the same. 
ness is defined as a layer in which the pores are of comparable size to the penetrating particles.

From the results described in this paper one can conclude that for PPO membranes the thickness of the impermeable layer, where gold particles are excluded, hardly depends on the size of the particles used (Fig. 3). Apparently, in PPO membranes a sharp transition exists between the (macro) pores of the support and the pores in the skin layer, with a size smaller than $6 \mathrm{~nm}$ in diameter. So for PPO the skin thickness is well defined as a layer where the pores are much smaller than the continuous pores in the sublayer. These results correspond with the thermoporometry measurements mentioned above indicating that PPO membranes can indeed be described by the simple model of asymmetric membranes, where skin and sublayer are supposed to have independent functions.

For PSf membranes the thickness of the region where particles are excluded increases when larger particles are used. This supports the idea that no discrete skin layer is present here and that the size of the pores gradually increases from skin layer to sublayer.

\section{Some Comments on the Blocking Mechanism}

The interpretations given are based on the direct relationship between pore size and particle size, as can be described by a simple sieving mechanism. In this conception the flow of particles is impeded by the difference in size of pores and particles only: a particle cannot penetrate into a pore of a size smaller than its own diameter. As is known from common ultrafiltration, transport through the membrane can be influenced by a number of other parameters like adsorption and pore blocking (1, $6,10)$. The situation described here differs from the normal UF setup. The membrane is permeated from the sublayer side, so the particles move from the large pores in the sublayer into smaller pores in the skin region. Forced by the pressure difference, the particles per- meate through the porous structure until the pores are too small and exclusion occurs when the pore size is smaller than the particle diameter. Actually, pore blocking is used here as an indication of the pore size present in the membrane.

\section{Possible Effects of Charges}

When colloids are ultrafiltered, coulombic forces between particles mutually and between particles and charged membrane walls may play a role too. The solid particles of a stable colloidal solution carry a definite positive or negative charge. The pore surface of the membrane material may also carry a charge, as is the case for practically every solid-liquid interface $(11,12)$. Because of the occurrence of these surface charges, either repulsive or attractive forces exist between the colloidal particles and the surface of the filter material.

When the interaction between particle and pore wall resulting from van der Waals or coulombic forces is positive, colloidal particles might be captured by the adhesion of the particles in the pores. The retention is then mainly caused by interaction between specific surface sites and particles $(11,13)$ and the size of the pore is of minor importance. If this mechanism prevails, the amount of particles possibly captured by the membrane is limited by the number of specific surface sites. By the time all the sites are occupied, retention can only be caused by differences in size of particles and constrictions. In deep-bed filtration, where the pore size of the filter used is much larger than the particles to be filtered, this phenomenon is known as breakthrough.

Measurements of the electrokinetic charge of colloidal gold particles have shown that the particles carry negative charges $(14,15)$. This was confirmed by electrophoretic measurements performed in our laboratory. These experiments were done using a simple Tiselius apparatus. It was observed that the interface between gold sol solution and its dialysate moved in the direction of the cathode, indi- 
cating that the particles were negatively charged. The zeta potential of the membrane materials under the conditions used here is expected to be negative $(1,19,20)$. As a result of these charges a repulsive force between pore wall and colloid particle exists, preventing the approach and adhesion of particles at the membrane wall. Since adhesion does not occur, the particles permeate through the porous structure until the pores and the particles are of comparable size.

Mutual interaction due to electrical double layer effects between the particles should be considered too. From literature on permeation of colloids it is known that, for example, during cake formation of filtered colloids, the structure of the cake is determined a great deal by the thickness of the double layer around the cake-forming particles (16-18). These effects are the result of particle-particle interaction for impermeable particles and the role of membrane structure is limited in that case.

The double layer thickness, however, might increase the effective radius of the particles which do penetrate into the pores, as we are studying. From the DLVO theory the dependence of double layer thickness $(1 / \kappa)$ on concentration of counterions is known $(14,18)$. For the sols that have been used here this thickness $\left(1 / \kappa=\left(10 c z^{2}\right)^{-0.5}\right.$, with $(1 / \kappa)$ in nanometers and where $c$ is the counterion concentration in moles/liter and $z$ is the valency of counterions; here $c=1.4 \times 10^{-3} \mathrm{~mol} /$ liter and $z=1$ ), is calculated to be about 10 $\mathrm{nm}$. Since the double layer is diffuse, it can be deformed very easily, as is known from electrophoretic experiments. Hence only a small part of the diffusive double layer codetermines the hydrodynamic behavior of the particle. The distance between the "hydrodynamic slip plane" and the particle is mainly determined by the size of the hydrated molecules adsorbed in the first layer around the sol particle. This increase in particle size is not more than a few tenths of a nanometer and the effective hydrodynamic size of the sol particles slightly differs from the values found by electron microscopy.
From this discussion it seems fair to assume that the colloidal gold particles are blocked only when the pore diameter and particle diameter are of comparable size. The impermeable layer consists of pores smaller than the size of the particles, whereas the pores at the boundary of the permeated and nonpermeated regions are of sizes comparable to those of the colloidal particles.

\section{CONCLUSION}

A new method for the determination of the skin thickness of UF membranes was developed. The method is based on the permeation of colloidal particles of well-known size entering from the macroporous sublayer side of an asymmetric UF membrane. The particles permeate through the large pores of the porous support until small pores near or in the skin are reached. In this way a thin layer is formed through which the colloidal particles cannot penetrate. This layer can be easily distinguished by scanning electron microscopy.

PPO and PSf membranes were investigated using this method. PPO membranes have a well-defined skin layer with a thickness of about $0.2 \mu \mathrm{m}$ and a pore size distinctly different from the pore size in the macroporous layer underneath. In case of PSf, a well-defined skin layer cannot be observed. The size of the pores in these membranes increases gradually from skin to sublayer.

\section{ACKNOWLEDGEMENT}

The authors acknowledge Dr. Ir. J. Beyer for performing the TEM measurements.

\section{REFERENCES}

1. Tragardh, G., in "Proceedings, Workshop on Characterization of Ultrafiltration Membranes" (G. Tragardh, Ed.), p. 9. Lund University, Lund, 1987.

2. Capanelli, G., Vigo, F., Munari, S., J. Membr. Sci. 15, 289 (1983).

3. Smolders, C. A., and Vugteveen, E., in "Material Science of Synthetic Membranes" (D. R. Loyd, Ed.), p. 327. ACS Symposium Series, Amer. Chem. Soc., Washington, DC, 1985.

4. Kamide, K., and Manabe, S., in "Ultrafiltration 
Membranes and Applications" (A. R. Cooper, Ed.), p. 173. Plenum, New York, NY, 1980.

5. Altena, F. W., Knoef, H. A. M., Heskamp, H., Bargeman, D., and Smolders, C. A., J. Membr. Sci. 12, 313 (1983).

6. Fane, A. G., and Fell, C. J. D., Desalination 62, 117 (1987).

7. Bargeman, D., Vugteveen, E., te Hennepe, J., van't Hof, J., and Smolders, C. A., in "Synthetic Polymeric Membranes" (B. Sedlacek and J. Kahovec, Eds.), p. 637, de Gruyter, Berlin, 1987.

8. Zsigmondy, R., Z. Anorg. Allg. Chem. 99, 105 (1917).

9. Frens, G., Kolloid Z. Z. Polym. 250, 73 (1972).

10. Fane, A. G., in "Progress in Filtration and Separation" (R. J. Wakeman, Ed.), Vol. 4, p. 101. Elsevier, Amsterdam, 1986.

11. Heertjes, P. M., and Lerk, C. F., Trans. Inst. Chem. Eng. 45, T129 (1967).

12. Cook, M. A., in "Hydrophobic Surfaces" (F. M.
Fowkes, Ed.), p. 206. Academic Press, New York, 1969.

13. Herzig, J. P., Leclerc, D. M., and le Cof, P., in "Flow through Porous Media" (R. J. Nunge, Ed.), p. 129. ACS Publications, Washington, DC, 1970.

14. Verwey, E. J. W., and Overbeek, J. Th. G., in "Theory of the Stability of Lyophobic Colloids." Elsevier, Amsterdam, 1948.

15. Glasstone, S., in "Textbook of Physical Chemistry" 2nd ed., p. 1239. McMillan, London, 1951.

16. Porter, M. C., Ind. Eng. Chem. Res. Dev. 11, 234 (1972).

17. McDonogh, R. M., Fane, A. G., and Fell, C. J. D., J. Membr. Sci. 21, 285 (1984).

18. Maron, S. H., and Lando, J. B., in "Fundamentals of Physical Chemistry" p. 788. Macmillan, New York, 1974.

19. Lee, C. K., and Hong, J., J. Membr. Sci. 39, 79 (1988).

20. Wei, Z., in "Proceedings, Workshop on Characterization of Ultrafiltration Membranes" (G. Tragardh, Ed.), p. 234. Lund University, Lund, 1987. 